\title{
Calcifications in Ovary and Endometrium and Their Neoplasms
}

Elvio G. Silva, M.D., Michael T. Deavers, M.D., A. F. Parlow, M.D., David M. Gershenson, M.D., Anais Malpica, M.D.

Departments of Pathology (EGS, AM, MTD) and Gynecologic Oncology (DMG), The University of Texas M. D. Anderson Cancer Center, Houston, Texas; and Department of Pathology (AFP), Harbor-UCLA

Medical Center, Torrance, California

In this study, we investigated the role of hormones in the pathogenesis of calcifications in ovary and in endometrium and their neoplasms of the gynecologic tract and assessed the anatomic location and incidence of these calcifications. The study consists of three parts designed to investigate the pathogenesis, the location, and the incidence of calcifications in ovary and endometrium and their neoplasms. In the first part, 79 female guinea pigs were divided into 10 groups, and different hormones, given weekly for 12 months, were administered to the guinea pigs by group. A control group of 7 guinea pigs received sterile water. Calcifications developed in 5 of 7 guinea pigs treated with prolactin, 10 of 20 treated with human chorionic gonadotropin, 5 of 11 treated with estradiol, 3 of 7 treated with estrone, 1 of 6 treated with growth hormone, and 1 of 10 treated with testosterone; in 20 of the guinea pigs, the calcifications developed in the stroma of the endometrium, and in $\mathbf{5}$ guinea pigs, they developed in the ovary. The second part of the study consisted of an evaluation of the specific location of calcifications in 43 consecutive human surgical ovaries and endometria. Calcifications were seen only in the stroma in $100 \%$ of the ovarian serous adenofibroma specimens; in ovarian serous borderline neoplasms, the stroma contained 70 to $100 \%$ of the calcifications, and the epithelium had 0 to $30 \%$ of the calcifications. In ovarian serous carcinoma specimens, the calcifications were seen in the stroma in $\mathbf{5 0}$ to $60 \%$ of the cases, in the epithelium in $40 \%$ of the cases, and in areas of necrosis in $10 \%$ of the cases.

Copyright (C) 2003 by The United States and Canadian Academy of Pathology, Inc.

VOL. 16, NO. 3, P. 219, 2003 Printed in the U.S.A.

Date of acceptance: January 8, 2003.

Supported in part by the Polo on the Prairie Grant Foundation and the Ovarian Cancer Research Program.

Address reprint requests to: Elvio G. Silva, M.D., Department of Pathology, Box 85, The University of Texas M. D. Anderson Cancer Center, 1515 Holcombe Boulevard, Houston, TX 77030; fax: 713-792-5529; e-mail: esilva@mdanderson.org.

DOI: 10.1097/01.MP.0000057236.96797.07
The third part of the study was directed to determine the frequency of calcifications in ovarian lesions. We found that all cases of endosalpingiosis and ovarian low-grade serous carcinoma had calcifications, whereas $80 \%$ of the cases of serous borderline tumor had calcifications, and only $50 \%$ of the cases of ovarian high-grade serous carcinoma contained calcifications. The results of this study indicate that the majority of the calcifications in the ovary and the endometrium and their neoplasms are present in the stroma. This is most probably secondary to metabolic changes, which could be related to hormones and not caused by degenerative changes in epithelial cells.

KEY WORDS: Calcifications, Endometrium, Hormones, Ovary, Pathogenesis, Tumor.

Mod Pathol 2003;16(3):219-222

Pathologic calcifications are classified as either metastatic (associated with hypercalcemia) or dystrophic (associated with normal calcemia) $(1,2)$. Traditionally, calcifications in neoplasms have been considered to be dystrophic, forming secondary to degeneration of the epithelium or in association with areas of necrosis (2-6). However, a secretory rather than a degenerative etiology has also been suggested on the basis of the absence of stainable DNA and RNA in areas with calcifications (7-9).

In this study, we investigated the role of hormones in the pathogenesis of calcifications in the ovary and the endometrium and their neoplasms and assessed the anatomic site and incidence of these calcifications. A novel pathogenetic mechanism to explain the presence of calcifications in the ovary and endometrium is presented.

\section{MATERIALS AND METHODS}

This study was divided into three parts to study the pathogenesis, the location, and the incidence of calcifications in the ovary and the endometrium 
and their neoplasms. To study the pathogenesis of calcifications, 79 female guinea pigs were divided into 10 different groups, and a different hormone was administered to each group. The hormones, the weekly doses and modes of administration, and the number of guinea pigs per group were as follows, respectively: estradiol, $1 \mathrm{mg}$ intramuscularly (IM), $n=11$; estrone, $1.2 \mathrm{mg} \mathrm{IM}, n=7$; testosterone, $80 \mathrm{mg}$ IM, $n=10$; dihydrotestosterone, $10 \mathrm{mg}$ orally, $n=4$; tamoxifen, 3 mg orally, $n=5$; megestrol acetate, $100 \mathrm{mg}$ orally, $n=4$; clomiphene, 1.5 mg orally, $n=5$; human chorionic gonadotropin, $500 \mathrm{U} \mathrm{IM}, n=20$; prolactin, $0.4 \mathrm{~mL} \mathrm{IM}, n=7$; and growth hormone, $0.2 \mathrm{~mL} \mathrm{IM}, n=6$. All of the hormones were administered for 12 months. The number of animals placed in each group was determined by our previous experience with these hormones $(10,11)$. Larger numbers of animals were placed in the groups receiving hormones likely to induce calcifications, whereas a smaller number of animals received the hormones that were less likely to induce calcifications, or hormones that were difficult to obtain, such as prolactin and growth hormone. The control group comprised 7 guinea pigs that received sterile water in doses of $2 \mathrm{~mL} / \mathrm{wk}$ IM for 12 months. After 12 months, all of the guinea pigs were killed, and a complete autopsy was performed on each.

To evaluate the locations of calcifications, we reviewed 43 consecutive human gynecologic specimens: 33 epithelial ovarian neoplasms, including 3 serous adenofibromas, 6 serous borderline tumors, 13 low-grade serous carcinomas, 10 high-grade serous carcinomas, and 1 undifferentiated carcinoma; and 10 endometria, including 5 proliferative endometrium, 3 endometrial hyperplasia, and 2 endometrial endometrioid adenocarcinomas with calcifications. We evaluated calcifications smaller than $30 \mu \mathrm{m}$ in greatest dimension because the precise origin of calcifications larger than $30 \mu \mathrm{m}$ generally could not accurately be determined. In specimens with small calcifications, we evaluated the location of the calcifications, the presence of necrosis or vascular changes, and the number of mitoses in the cells near the calcifications.

To assess the frequency of calcifications, we investigated the presence or absence of calcifications in a retrospective review of 56 serous lesions, including 10 cases of ovarian endosalpingiosis and 46 cases of ovarian neoplasms (10 serous borderline tumors, 16 low-grade serous carcinomas, and 20 high-grade serous carcinomas).

The low- and high-grade serous carcinomas were classified by criteria recently proposed by Malpica et al. (12). Serous carcinomas with $\leq 10$ mitoses per 10 high-power fields or absence of significant nuclear pleomorphism were classified as low-grade tumors, and those with $>10$ mitoses per 10 highpower fields or with significant nuclear pleomorphism were classified as high-grade tumors.

\section{RESULTS}

Calcifications were found in 25 of the 79 guinea pigs that received hormones. No calcifications were found in the guinea pigs that received sterile water. The number of guinea pigs with calcifications, by the hormone received, was as follows: estradiol, 5 of 11 guinea pigs; estrone, 3 of 7 guinea pigs; testosterone, 1 of 10 guinea pigs; dihydrotestosterone, 0 of 4 guinea pigs; tamoxifen, 0 of 5 guinea pigs; megestrol, 0 of 4 guinea pigs; clomiphene, 0 of 5 guinea pigs; human chorionic gonadotropin, 10 of 20 guinea pigs; prolactin, 5 of 7 guinea pigs; and growth hormone, 1 of 6 guinea pigs. The calcifications were found in the stroma of the endometrium and within endometrial glands in 20 of the 25 cases, and in the ovarian stroma in the remaining 5 cases. Each of these 25 cases had one to five foci containing calcifications and had no areas of necrosis associated with these calcifications.

In the 43 gynecologic specimens reviewed to determine the location of the calcifications, the sites varied by the histologic type of lesion. Table 1 shows the results of the 33 ovarian neoplasms. In the three ovarian serous adenofibromas, the calcifications were present only in the stroma. Necrosis and mitoses were not seen in these areas. In the six ovarian serous borderline tumors, the percentage of calcifications in each tumor present in the epithelium ranged from 0 to $30 \%$ (median, $0 \%$ ); conversely, the percentage in the stroma ranged from 100 to $70 \%$ (median, 100\%; Fig. 1). No necrosis or

TABLE 1. Histologic Features, Distribution, and Frequency of Calcifications in 33 Human Epithelial Ovarian Neoplasms

\begin{tabular}{|c|c|c|c|c|c|}
\hline \multirow[b]{2}{*}{ Histologic Diagnosis Number of Cases (n) } & \multicolumn{4}{|c|}{ Percentage of Calcifications by Site, Range (Median) } & \multirow{2}{*}{$\begin{array}{c}\text { Number of } \\
\text { Mitoses per } 10 \\
\text { HPF, Median }\end{array}$} \\
\hline & Epithelium & Stroma & $\begin{array}{c}\text { Area of } \\
\text { Necrosis }\end{array}$ & Necrosis & \\
\hline Serous adenofibroma, $n=3$ & $0(0)$ & $100(100)$ & - & - & 0 \\
\hline Serous borderline, $n=6$ & $0-30(0)$ & $70-100(100)$ & - & - & 0 \\
\hline Low-grade serous carcinoma, $n=13$ & $10-60(40)$ & $40-80(60)$ & $5-10(7)$ & + & 3 \\
\hline $\begin{array}{l}\text { High-grade serous carcinoma, } n=10 \\
\text { and undifferentiated carcinoma, } n=1\end{array}$ & $20-70(37)$ & $30-80(50)$ & $4-40(10)$ & +++ & 21 \\
\hline
\end{tabular}

Necrosis: $+=$ one focus $<3 \mathrm{~mm} ;++=$ two to three foci, $<3 \mathrm{~mm} ;+++=$ over three foci or larger than $3 \mathrm{~mm}$. 


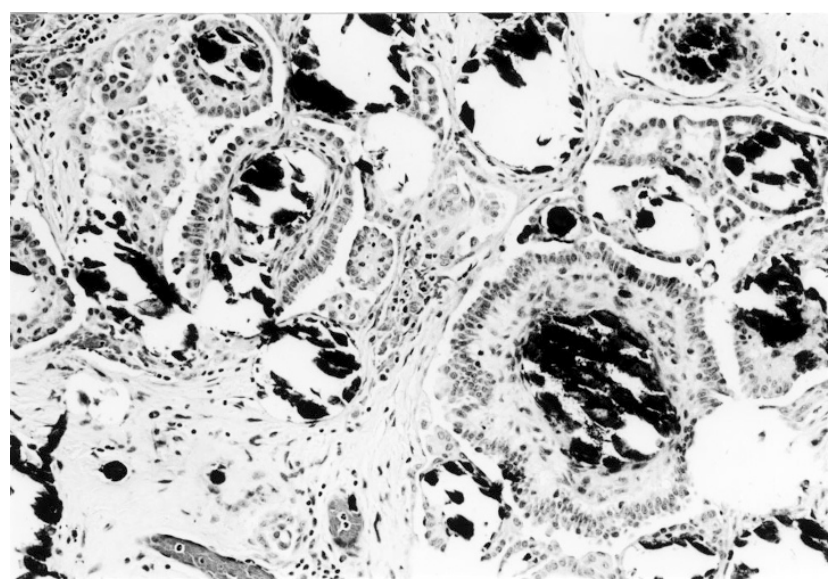

FIGURE 1. Ovarian serous borderline tumor. Small calcifications in the stroma; several large calcifications at the center of the papillae.

mitoses were present in these areas. In the 13 ovarian low-grade serous carcinomas, the percentage of the calcifications in the epithelium ranged from 10 to $60 \%$ (median, $40 \%$ ), and the percentage in the stroma ranged from 80 to $40 \%$ (median, $60 \%$; Fig. 2). In a single case of ovarian low-grade serous carcinoma, $10 \%$ of the calcifications were in an area of necrosis. One to seven (median, 3) mitotic figures per 10 high-power fields were present in these specimens. In the 10 ovarian high-grade serous carcinomas and in the only ovarian undifferentiated carcinoma, the percentage of calcifications in the epithelium varied from 20 to $70 \%$ (median, $37 \%$; Fig. 3), the percentage of calcifications in the stroma ranged from 30 to $80 \%$ (median, $50 \%$ ), and the percentage of calcifications in areas of necrosis ranged from 4 to $40 \%$ (median, $10 \%$ ). Six of the 10 cases of high-grade serous carcinoma and the undifferentiated carcinoma had areas of necrosis. In this group, the number of mitotic figures ranged from 10 to 30 (median, 21) per 10 high-power fields.

In the 10 cases with endometrial calcifications, the location of the calcifications varied by the diagnosis.

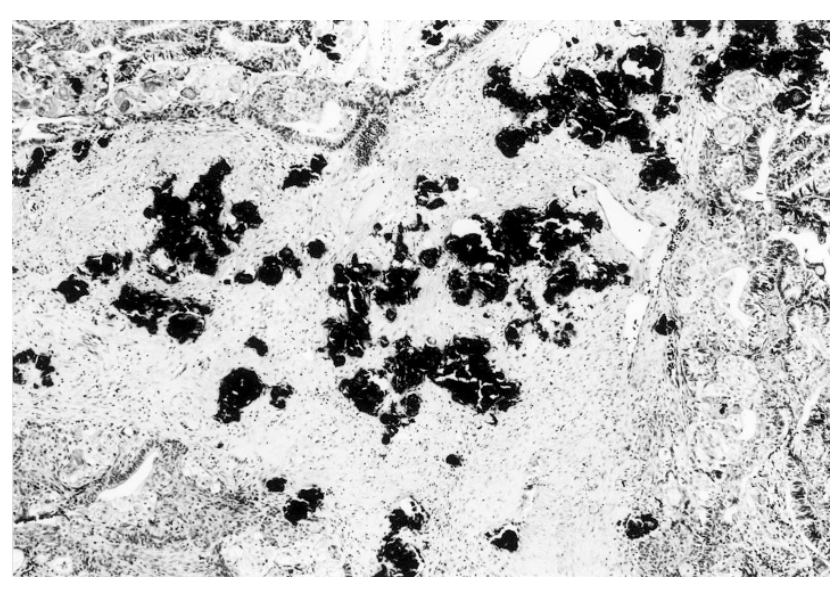

FIGURE 2. Ovarian carcinoma. Multiple calcifications in an area devoid of epithelium.

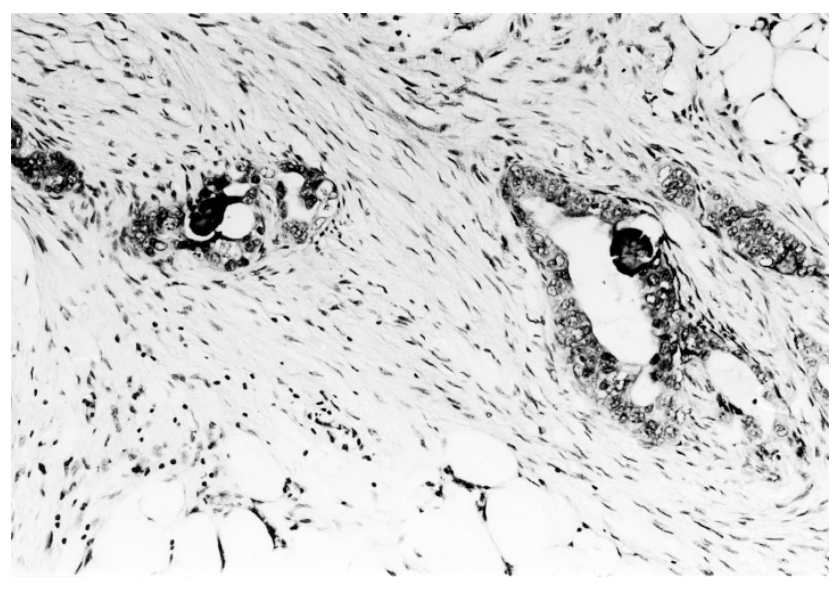

FIGURE 3. Serous carcinoma in omentum. Calcifications in the epithelium of glands.

In the cases of proliferative endometrium, $90 \%$ of the calcifications were in the stroma, and $10 \%$, within glands. In the cases of simple endometrial hyperplasia, $100 \%$ of the calcifications were in the lumens of the glands; and in the cases of endometrial adenocarcinoma, $70 \%$ of the calcifications were in the epithelium and $30 \%$ were in the stroma. No vascular changes were seen in any of the 43 cases reviewed.

In our assessment of 56 cases with the objective of determining the frequency of calcifications, we found calcifications in all 10 cases of endosalpingiosis and all 15 ovarian low-grade serous carcinomas. Eight of the $10(80 \%)$ ovarian serous borderline neoplasms and 10 of the 20 (50\%) ovarian highgrade serous carcinomas had calcifications. In most cases, the calcifications were scattered and focal, and large areas without calcifications were seen between foci with calcifications.

\section{DISCUSSION}

The most widely accepted theory regarding the pathogenesis of calcifications postulates that calcifications are due to calcium deposition in areas of cellular degeneration associated with either an infectious process, such as that which occurs in malakoplakia, or with ischemic changes, such as those seen in papillary neoplasms (2-6). It has been proposed that in ovarian serous neoplasms, calcifications start in the epithelial cells and stromal histiocytes. This theory has been questioned, however, by some investigators who have proposed that the calcifications might be related to a secretory phenomenon (7-9). This hypothesis is based on the absence of DNA and RNA and the presence of acid mucopolysaccharides in the areas of calcification.

Calcifications have also been described in various neoplasms associated with hormone production, including duodenal somatostatinoma, carcinoid tumor, prolactinoma, calcifying Sertoli cell tumor, 
and gonadoblastoma, and in benign endometria of patients receiving Enovid for contraception and clomiphene (13-16).

In this study, we observed that calcifications can be induced in guinea pigs by hormone administration. Calcifications were identified in $71 \%$ of the guinea pigs given prolactin, $50 \%$ of those given human chorionic gonadotropin, $45 \%$ of those given estradiol, $43 \%$ of those given estrone, $17 \%$ of those given growth hormone, and $10 \%$ of those given testosterone. Therefore, we conclude that calcifications are not induced by only one hormone or a specific group of hormones.

In our assessment of the locations of calcifications in the ovary and endometrium, the incidence of calcifications was higher in the stroma than in the epithelium; however, the incidence varied by type of the lesion. In serous adenofibroma, calcifications were present only in the stroma; in borderline serous tumors, 70 to $100 \%$ of the calcifications were present in the stroma. In carcinoma, 40 to $80 \%$ of the calcifications were seen in the stroma, 10 to $70 \%$ in the epithelium, and the rest in areas of necrosis. In the 5 cases of proliferative endometrium, $90 \%$ of the calcifications were in the stroma and $10 \%$ in the lumen of the glands. In the cases of endometrial hyperplasia and adenocarcinoma, most of the calcifications were in the lumen of the glands. The higher frequency of calcifications in the stroma suggests that calcifications are not associated with degenerative epithelial changes. In epithelial tumors, the epithelial cells have a higher proliferation rate and are farther away from the vessels than are the stromal cells, which would make the epithelial cells more prone to degenerative changes. Although we do not know why calcifications occur predominantly in the stroma, the phenomenon could be related to metabolic changes secondary to paracrine effects in the stromal cells. Our review also showed that even in tumors with significant necrosis, only 4 to $40 \%$ (median, 10\%) of the calcifications occurred in necrotic areas.

In the cases reviewed to study the frequency of calcifications, we found that all 10 cases of endosalpingiosis, and all 15 ovarian low-grade serous carcinomas had calcifications, whereas only $80 \%$ of the ovarian serous borderline tumors and $50 \%$ of ovarian high-grade serous carcinomas had calcifications. The presence of calcifications in all cases of benign conditions and low-grade neoplasms and in only $50 \%$ of the high-grade carcinomas also supports the theory that calcifications in ovary and endometrium and their neoplasms are rarely due to cellular degeneration. If calcifications are mainly caused by cellular degeneration, then they should occur more frequently in high-grade neoplasms than in benign conditions.

Psammocarcinoma is composed almost entirely of calcifications, with very few epithelial cells (17). It would be impossible to explain psammocarcinoma as a lesion caused by calcification of epithelial cells.

In summary, we have demonstrated that calcifications in the ovary and endometrium and their neoplasms can be induced by various hormones in guinea pigs and that they are more frequently seen in the stroma, especially in benign and low-grade ovarian human tumors. It is likely that multiple mechanisms are involved in the pathogenesis of calcifications, because calcifications are also seen in areas of necrosis and in tumors with degenerative changes. Understanding the pathogenesis of calcifications might have an impact on the development of new diagnostic and therapeutic modalities.

\section{REFERENCES}

1. Cotran RS. Cellular pathology II: adaptations, intracellular accumulations, and cell aging. In: Robbins pathologic basis of disease. 6th ed. Philadelphia: Saunders; 1992. p. 31-49.

2. Walter JB, Talbot IC. Calcium metabolism and heterotopic calcification. Walter and Israel general pathology. 7th ed. New York: Churchill Livingstone; 1996. p. 635-49.

3. Factor SM. Papillary adenocarcinoma of the endometrium with psammoma bodies. Arch Pathol 1974;98:201-5.

4. Ferenczy A, Talens M, Zoghby M, Hussain SS. Ultrastructural studies on the morphogenesis of psammoma bodies in ovarian serous neoplasia. Cancer 1977;39:2451-9.

5. Cribbs RK, Ishaq M, Arnold M, O’Brien J, Lamb J, Frankel WL. Renal cell carcinoma with massive osseous metaplasia and bone marrow elements. Ann Diagn Pathol 1999;3:294-9.

6. Seltzer V, Spitzer M. Psammoma bodies in papillary adenocarcinoma of the endocervix. Int J Gynecol Pathol 1983;2:216-21.

7. Karpas CM, Bridge MF. Endometrial adenocarcinoma with psammoma bodies. Am J Obstet Gynecol 1963;87:935-41.

8. Ahmed A. Calcification in human breast carcinomas: ultrastructural observations. J Pathol 1975;117:247-50.

9. Groisman GM, Amar M, Polak-Charcon S. Microcalcifications in the anterior pituitary gland of the fetus and the newborn: a histochemical and immunohistochemical study. Hum Pathol 1999;30:199-202.

10. Silva EG, Tornos C, Fritsche HA Jr, El-Naggar A, Gray K, Ordonez NG, et al. The induction of benign epithelial neoplasms of the ovaries of guinea pigs by testosterone stimulation. A potential animal model. Mod Pathol 1997;10:879-83.

11. Silva EG, Tornos C, Deavers M, Kaisman K, Gray K, Gershenson DM. Induction of epithelial neoplasms in the ovaries of guinea pigs by estrogenic stimulation. Gynecol Oncol 1998;71:240-6.

12. Malpica A, Deavers MT, Lu K, Liu J, Atkinson EN, Gershenson DM, et al. Grading ovarian serous carcinomas using a two-tier system. Abstract Mod Pathol 2002;15:814.

13. Radaković; B, Jukić; S, Buković; D, Ljubojević; N, Čima I. Morphology of gonads in pure XY gonadal dysgenesis. Coll Antropol 1999;23:203-11.

14. Valicenti JF Jr, Priester SK. Psammoma bodies of benign endometrial origin in cervicovaginal cytology. Acta Cytol 1977;21:550-2.

15. Albrecht S, Gardiner GW, Kovacs K, Ilse G, Kaiser U. Duodenal somatostatinoma with psammoma bodies. Arch Pathol Lab Med 1989;113:517-20.

16. Herbold DR, Magrane DM. Calcifications of the benign endometrium. Arch Pathol Lab Med 1986;110:666-9.

17. Gilks CB, Bell DA, Scully RE. Serous psammocarcinoma of the ovary and peritoneum. Int J Gynecol Pathol 1990;9:110-2. 